\title{
Biometria e eficiência do uso da água em tomate cereja no semiárido
}

\author{
Magna Soelma Beserra de Moura ${ }^{1(*)}$, Luciana Sandra Bastos de Souza ${ }^{2}$, Leide Dayane da Silva Oliveira ${ }^{3}$, \\ Thieres George Freire da Silva ${ }^{2}$, Jony Eishi Yuri ${ }^{1}$ \\ ${ }^{1}$ Pesquisador(a), Embrapa Semiárido, Petrolina, PE, magna.moura@embrapa.br, jony.yuri@embrapa.br \\ ${ }^{2}$ Professor(a), UFRPE/UAST, Serra Talhada, PE, sanddrabastos@hotmail.com, thigeoprofissional@hotmail.com \\ ${ }^{3}$ Bolsista BFT/FACEPE, Embrapa Semiárido, Petrolina, PE, leide_dayane2014@hotmail.com \\ ${ }^{(*)}$ Autor para correspondência
}

\section{INFORMAÇÕES}

\section{História do artigo:}

Recebido em 16 de Junho de 2017

Aceito em 10 de agosto de 2017

Termos para indexação:

crescimento

produtividade

requerimento hídrico

Solanum lycopersicum
RESUMO

O objetivo deste trabalho foi avaliar a biometria e eficiência do uso da água em tomate cereja no Semiárido. O experimento foi conduzido em duas épocas do ano, com ciclos iniciados em 12 de Setembro de 2012 e 15 de Abril de 2013, no campo experimental de Bebedouro na Embrapa, Petrolina-PE. As cultivares avaliadas foram a F1170, Abirú, Tanzimech e E5663, nas quais monitorou-se o diâmetro do caule, comprimento do ramo principal e número de folhas expandidas, ao longo do ciclo, e na ocasião da colheita, foi mensurada a produtividade. Na análise da eficiência do uso da água foram usados os indicadores produtividade da água da cultura (PAC, $\mathrm{kg} \mathrm{m}^{3-}$ ) e a produtividade econômica da água (PEA, $\mathrm{R} \$ \mathrm{~m}^{-3}$ ). Os resultados mostraram que, na segunda época de plantio (15 de Abril de 2013), o crescimento do tomate cereja foi superior para a cv. Tanzimech $(214,00 \mathrm{~cm})(\mathrm{p}<0,05)$. Todavia, a maior produtividade $\left(75,1\right.$ toneladas $\left.h^{-1}\right)$, eficiência do uso da água $\left(37,89 \mathrm{Kg} \mathrm{m}^{3-}\right)$ e produtividade econômica da água $\left(242,88 \mathrm{R} \$ \mathrm{~m}^{-3}\right)$ foram superiores para a cv. E5663, sugerindo que seu cultivo seja incentivado nas condições do Submédio do Vale do São Francisco.

(c) 2017 SBAgro. Todos os direitos reservados.

\section{Introdução}

O cultivo do tomate cereja tem apresentado um crescimento expressivo, em decorrência, principalmente, das suas propriedades organolépticas e da grande aceitabilidade pela população (LEONARDI et al., 2000; LENUCCI et al. 2006). Estudos têm sido conduzidos com a finalidade de analisar as respostas da cultura do tomate quanto à eficiência de uso da radiação (RADIN et al., 2003), utilização de nutrientes (ARAÚJO et al., 2013, OLIVEIRA et al., 2009) e produção (SARI et al., 2017, AGUIRRE; CABRERA, 2012, GUSMÃO; GUSMÃO; ARAÚJO, 2006, CARVALHO; TESSARIO-
LI NETO, 2005). Entretanto, ainda não se têm estudos sobre o crescimento e as respostas produtivas desta cultura nas condições climáticas do Semiárido Brasileiro. Os estudos realizados geralmente são direcionados ao comportamento produtivo do tomate salada, sendo mais escassos para o tomate cereja (SOUZA; MEDEIROS; CARNEIRO, 2010).

O crescimento das plantas é particularmente sensível às condições ambientais, de modo que indicadores do desenvolvimento da parte aérea e de produção têm sido utilizados com o objetivo de inferir a influência de fatores bióticos e abióticos no rendimento final das culturas (KUMAR et al., 2006, LIU; STUTZEL, 2004, PAYERO et al., 2008). Outra 
forma de analisar as resposta dos cultivos ao ambiente é por meio do cômputo dos indicadores de eficiência do uso de água (PEREIRA; CORDERY; IACOVOS, 2012). Dentre estes pode-se citar a produtividade de água da cultura (PAC) que relaciona a produção de biomassa seca e, ou, produção comercial, pela quantidade de água aplicada ou evapotranspirada (LIU; STUZEL, 2004; PUPPALA et al., 2005) e a produtividade econômica da água, que permite mensurar o incremento produtivo e o retorno econômico bruto em função do uso de água no sistema de produção (PEREIRA; CORDERY; IACOVOS, 2012).

Estudos dessa natureza não foram amplamente realizados para o tomate cereja, fortalecendo a necessidade de sua execução visando melhorar a compreensão das respostas das plantas à disponibilidade de água sob as condições climáticas do Semiárido brasileiro. Assim, o objetivo deste trabalho foi avaliar o crescimento, produtividade e a eficiência do uso da água de cultivares de tomate cereja sob as condições do Submédio do Vale do São Francisco.

\section{Material e métodos}

\section{Caracterização da área experimental}

O experimento foi conduzido no Campo Experimental de Bebedouro (0909'S; 40²2'W e 365,5m), na Embrapa Semiárido, Petrolina-PE, onde foram instalados dois ensaios, sendo o primeiro entre os meses de agosto e dezembro de 2012, e o segundo entre os meses de março e julho de 2013. o clima da região segundo a classificação de Köppen é do tipo BSwh', ou seja, Semiárido com temperaturas do ar médias anuais elevadas, da ordem de $26^{\circ} \mathrm{C}$, e precipitação média de 514,7 mm (EMBRAPA SEMIÁRIDO, 2013). O solo da área experimental (classificado como Podzólico Amarelo eutrófico latossólico) foi previamente analisado quimicamente, apresentando os seguintes resultados: $\mathrm{P}=51,43$ $\mathrm{mg} \mathrm{dm}{ }^{-3} ; \mathrm{K}=1,30 \mathrm{cmol}_{\mathrm{c}} \mathrm{dm}^{-3} ; \mathrm{Ca}=0,70 \mathrm{cmol}_{\mathrm{c}} \mathrm{dm}^{-3} ; \mathrm{Mg}=0,40$ $\mathrm{cmol}_{\mathrm{c}} \mathrm{dm}^{-3} ; \mathrm{H}+\mathrm{Al}=2,24 \mathrm{cmol}_{\mathrm{c}} \mathrm{dm}^{-3} ; \mathrm{CTC}$ efetiva $=4,73 \mathrm{cmol}_{\mathrm{c}}$ $\mathrm{dm}^{-3} ; \mathrm{V} \%=52,64 ; \mathrm{pH}$ em água $(1: 2,5)=6,78 ; \mathrm{B}=0,90 \mathrm{mg} \mathrm{dm}^{-3}$; $\mathrm{Cu}=0,80 \mathrm{mg} \mathrm{dm}^{-3} ; \mathrm{Fe}=14,90 \mathrm{mg} \mathrm{dm}^{-3} ; \mathrm{Mn}=41,30 \mathrm{mg} \mathrm{dm}^{-3}$; $\mathrm{Zn}=3,50 \mathrm{mg} \mathrm{dm}^{-3}$.

O preparo de solo, nas duas épocas de plantio, constou de aração, gradagem e confecção dos canteiros. Posteriormente, de posse da análise de solo, foi realizada a adubação de fundação, de acordo as recomendações de Cavalcante (2008), incorporando-o até uma profundidade de 0,1 m, dentro do limite vertical do canteiro. Em cada canteiro foi instalada uma linha de tubo gotejador com emissores com vazão de 1,6 L h ${ }^{-1}$, diâmetro nominal (DN) de $16 \mathrm{~mm}$ e espaçados entre si de 0,50 m. Em seguida, aplicou-se um filme plástico (mulching) na cor prata sobre os canteiros, que foi furado com um tubo de $75 \mathrm{~mm}$ a cada $0,5 \mathrm{~m}$, resultando em espaçamento entrelinhas e entre plantas foi de 2,0 m x 0,5 m, respectivamente. Dentre os tratos culturais, foram realizadas capinas (entre os canteiros e nos orifícios do mulching, ao redor do tomateiro) e aplicações de fungicidas e inseticidas necessárias à adequada condução dos trabalhos, livres de plantas invasoras, pragas e doenças.

Os tratamentos da primeira e segunda época de plantio foram compostos de quatro cultivares de tomate cereja (F1170, Abirú, Tanzimech e E5663). No primeiro, a semeadura foi realizada em 14 de agosto de 2012, em bandejas de isopor, e as mudas transplantadas em 11 de setembro de 2012. Já para o segundo experimento, a semeadura ocorreu em 19 de março de 2013 e as mudas transplantadas em 15 de abril de 2013. Adotou-se o delineamento experimental em blocos ao acaso, com quatro repetições. Cada parcela experimental apresentava a dimensão de 7,00 $\mathrm{m}^{2}(2,00 \mathrm{x}$ $3,50 \mathrm{~m})$, desconsiderando a bordadura. Para ambos os ensaios, as cultivares utilizadas foram de crescimento determinado, sendo conduzidas sem tutoramento.

Os dados referentes às condições meteorológicas da região foram obtidos por meio de uma estação agrometeorológica, localizada a cerca de $300 \mathrm{~m}$ da área experimental. A mesma foi equipada com sensores eletrônicos acoplados a um sistema automático de aquisição de dados datalloger (CR10X, Campbell Scientific) programado para realizar leituras a cada dez segundos e armazenar médias de trinta minutos dos seguintes elementos meteorológicos: temperatura e umidade relativa do ar, precipitação, radiação solar global e velocidade do vento.

\section{Dados biométricos}

As medições biométricas do primeiro e segundo ensaio foram iniciadas dois dias após o transplante das mudas estabelecendo-se o intervalo de aproximadamente 10 e 15 dias, o que resultou em doze e oito amostragens realizadas durante os dois ciclos produtivos, respectivamente. Nesses momentos foram avaliados os seguintes parâmetros: comprimento da haste principal (COM), diâmetro médio do caule (DMC) e número de folhas verdes completamente expandidas (NFVCE). No primeiro ensaio, os dados biométricos foram monitorados apenas as cultivares 'F1170', 'Abirú' e 'Tanzimech', enquanto no segundo experimento, essas mensurações foram adicionadas a cultivar cv. E5663.

o COM foi obtida por meio de uma trena, medindo-se do nível do solo até a primeira ramificação do caule principal. O DMC foi determinado a três centímetros de altura do solo, com auxílio de um paquímetro. O NFVCE foi contado em cada uma das ramificações da planta, sendo consideradas folhas verdes aquelas que apresentavam pelo menos $50 \%$ de área foliar com coloração verde. Concomitantemente, foram realizadas visitas diárias aos experimentos para delimitação das datas de ocorrência dos eventos fenológicos da cultura. Estes, por sua vez, foram expressos tanto em função dos dias decorridos após o transplantio (DAT), como em função dos graus-dia acumulados (GDA). 
Os GDA, ou exigência térmica do tomate, foram calculados assumindo a temperatura base de $10^{\circ} \mathrm{C}$ e a equação proposta por Ometto (1981), como segue:

$$
G D A=\sum t m-t b
$$

em que, $t_{m}=$ temperatura média e $t_{b}=$ temperatura base .

O NFVCE foi relacionado com os graus-dia acumulados para determinação da taxa de aparecimento foliar (folha $\left({ }^{\circ} \mathrm{C} \text { dia }\right)^{-1}$ ) por meio do coeficiente angular da regressão linear. O filocrono $\left({ }^{\circ} \mathrm{C}\right.$ dia folha $\left.{ }^{-1}\right)$ foi obtido invertendo-se a taxa de aparecimento foliar.

\section{Indicadores de eficiência produtiva do tomateiro}

As colheitas do primeiro e segundo experimento ocorreram, respectivamente, entre 8 de novembro e 18 de dezembro de 2012, e de 21 de junho a 24 de julho de 2013. o procedimento adotado para análise do rendimento das plantas foi o mesmo para todas as cultivares, compreendendo a coleta de três plantas da parcela útil de cada tratamento, de onde foram retirados os frutos, que foram quantificados e pesados para a determinação do rendimento total.

Com esses dados também foram calculados os indicadores para analisar a eficiência do uso de água das diferentes cultivares: a produtividade de água da cultura (PAC, kg $\mathrm{m}^{3-}$ ) e a produtividade econômica da água (PEA) (PEREIRA; CORDERY; IACOVOS, 2012). A produtividade de água da cultura foi calculada relacionando a produção de frutos (PF) e a lâmina de água aplicada (LAA), utilizando a eq. 2 (GEERTS; RAES, 2009; LACERDA et al., 2009). Já a produtividade econômica da água foi obtida pela relação entre a produção da cultura e o preço da mesma no mercado (média dos últimos cinco anos) (eq. 3).

$$
P A C=\frac{P F}{L A A}
$$

em que, PF é a produção de frutos ( $k g$ ha $\left.{ }^{1^{-}}\right)$e LAA corresponde a lâmina de água aplicada $\left(\mathrm{m}^{3} \mathrm{ha}^{{ }^{1-}}\right)$.

$$
P E A=\frac{G E}{L A A}
$$

em que, GE é o ganho econômico por unidade de área, em $\mathrm{R} \$ \mathrm{ha}^{-1}$, que considerará o preço médio do tomate cereja no mercado, nos últimos sete anos (CEASA-BA, 2013).

Para os dados obtidos, foram realizadas correlações entre as variáveis biométricas com dados de graus-dia acumulados, para as quais foram ajustadas regressões com o objetivo de caracterizar o crescimento das diferentes cultivares do tomate cereja na região Semiárida em cada ciclo.
Os dados obtidos ao longo do período experimental também foram avaliados estatisticamente por meio da análise de variância - ANOVA, sendo que os efeitos individuais dos tratamentos foram obtidos pelo teste de Tukey, ao nível de $5 \%$ de probabilidade, por meio da utilização do Software Sisvar.

\section{Resultados e discussão}

\section{Condições meteorológicas}

Constatou-se que o primeiro ciclo (Ciclo 1) foi conduzido em um período com temperatura do ar, variando entre 23,4 e $31,1^{\circ} \mathrm{C}$, com média de $28,1^{\circ} \mathrm{C}$ (Figura 1a), enquanto o segundo ciclo (Ciclo 2) transcorreu sob temperaturas mais amenas, oscilando de 20,9 a $30,0^{\circ} \mathrm{C}$, com média em torno de $24,5^{\circ} \mathrm{C}$ (Figura 1a). Estes valores estiveram dentro da faixa ótima para o desenvolvimento do tomateiro, os quais de acordo com Alvarenga (2004), situam-se entre 10 e $34^{\circ} \mathrm{C}$. Comportamento inverso ao da temperatura do Ciclo 1 foi observado para a umidade relativa do ar (Figura 1b), que apresentou valores baixos, com média de $48,8 \%$ e valores máximos e mínimos de 70,5 e 34,7\%, respectivamente. Já a umidade relativa do ar do Ciclo 2 foi, em média, igual a 61,2\%, com máximos e mínimos de 81,5 e 39,2\%, respectivamente. Tais valores estão associados à época de condução dos experimentos, uma vez que o Ciclo 2 transcorreu no primeiro semestre, quando ocorrem as maiores precipitações na região, enquanto que o Ciclo 1 foi conduzido no segundo semestre do ano, climaticamente caracterizado como o período seco na região de estudo.

No primeiro ciclo ocorreram eventos de chuva com lâmina inferior a 7,0 $\mathrm{mm} \mathrm{dia}{ }^{-1}$, concentrados entre $50 \mathrm{e}$ 60 dias após o transplantio (DAT) (Figura 1d); já no Ciclo 2, a precipitação ocorreu tanto no início do ciclo (até os 20 DAT) quanto no final, com valores diários inferiores a 6,0 mm (Figura 1b). o total de precipitação observado no Ciclo 1 foi de $12,19 \mathrm{~mm}$, enquanto no Ciclo 2, choveram 30,93 mm, ou seja, menos que os registros históricos para a região conforme Teixeira (2010). A radiação solar média atingiu valores em torno de 19,95 e 14,31 $\mathrm{MJ} \mathrm{m}^{-2} \mathrm{dia}^{-1}$, para os ciclos 1 e 2 , respectivamente, enquanto em alguns dias verificaram-se valores mínimos da ordem de 8,69 e 4,69 MJ m ${ }^{-2}$ dia $^{-1}$ e máximos de 27,67 e 24,26 MJ m${ }^{-2}$ dia $^{-1}$ (Figura 1c). Segundo Andriolo (2000), para o tomate, o nível de radiação solar global em que a planta produz o mínimo de fotoassimilados necessários à sua manutenção é de aproximadamente 8,4 $\mathrm{MJ} \mathrm{m}^{-2} \mathrm{dia}^{1-}$, e conforme (Figura 1c), apenas em dois dias do Ciclo 2 a radiação solar global incidente esteve abaixo desse valor, o que certamente não interferiu nos atributos de crescimento e produção do tomate.

Biometria do tomate cereja 
O diâmetro do caule a 3,0 cm de altura do solo (DMC) para diferentes cultivares é demonstrado em função dos
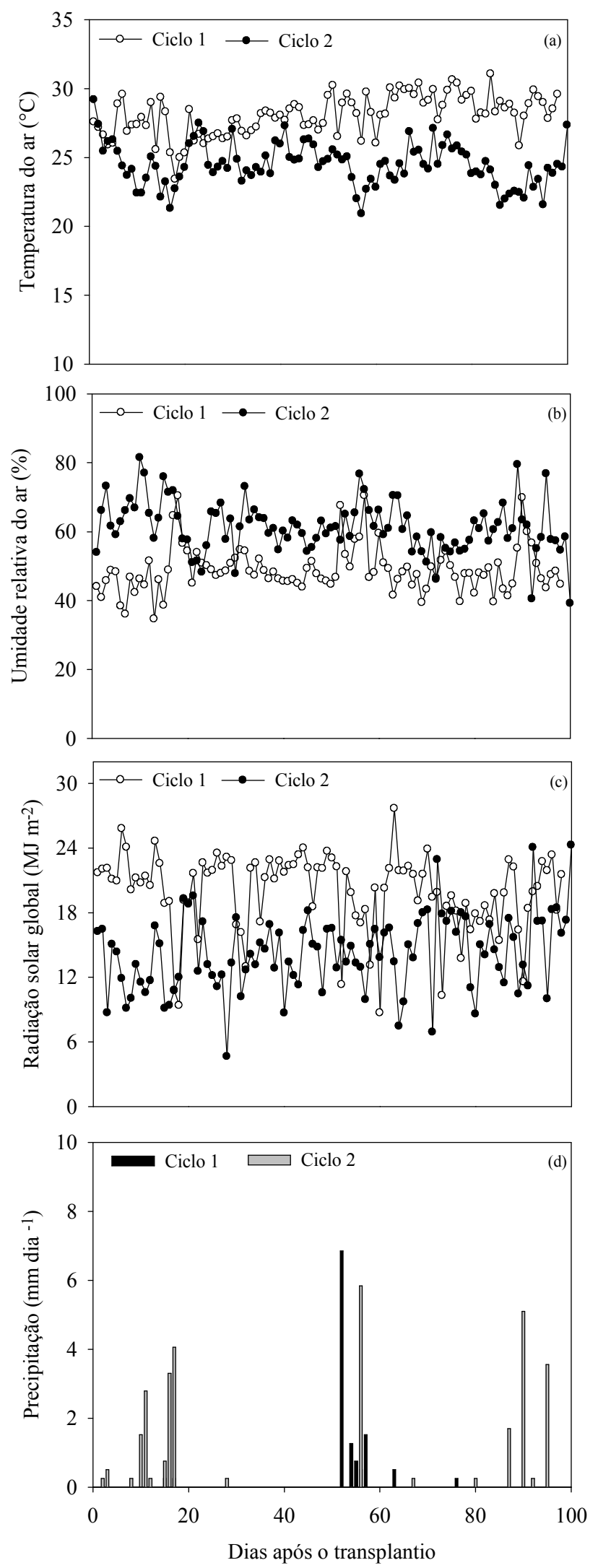

Figura 1. Variáveis meteorológicas durante o período de execução do experimento (Ciclo 1 e Ciclo 2) de tomate cereja em Petrolina-PE: (a) temperatura do ar, (b) umidade relativa do ar; (c) radiação solar global; (d) precipitação. graus dias acumulados para o primeiro (Ciclo 1) e o segundo (Ciclo 2) ciclo da cultura do tomate cereja (Figura 2). Observou-se que no início de ambos os ciclos, quando a cultura apresentou crescimento lento, as cultivares exibiram caules com diâmetro bastante semelhantes, variando em torno de $0,2 \mathrm{~cm}$ e $0,3 \mathrm{~cm}$, respectivamente no Ciclo 1 (Figura 2a) e Ciclo 2 (Figura 2b). Em ambos os ciclos, quando a cultura acumulou em torno de 700 e 500 graus dias pode-se constatar uma fase de crescimento rápido que, provavelmente, esteve associada à proximidade da fase reprodutiva da espécie. Neste período ocorreu um aumento na massa foliar da cultura, que exige uma maior rigidez do caule a fim de suportar o peso da copa. Ao final, por ocasião da colheita, no Ciclo 1, a cultivar Abirú se sobressaiu em relação às demais com maior diâmetro de caule $(3,37 \mathrm{~cm})(\mathrm{p}<0,05)$, seguida da 'F1170' $(3,35 \mathrm{~cm})$ e da 'Tanzimech' $(2,89 \mathrm{~cm})$ (Figura 2a). Para o Ciclo 2, a cultivar cv. E5663 sobressaiu com diâmetro em torno de $3,07 \mathrm{~cm}$, seguida da 'Abirú' $(2,60 \mathrm{~cm})$, da 'F1170' (2,47 cm) e da 'Tanzimech' (2,10 cm) (Figura 2b).

Gomes Júnior et al. (2011) analisando o tomateiro do grupo cereja observaram valores do diâmetro do caule em torno de 1,38 cm; enquanto Araújo et al. (2013) obtiveram valores menores, em torno de $0,75 \mathrm{~cm}$. As diferenças encontradas e o fato de ter sido observado um maior diâmetro do caule no presente estudo podem estar relacionadas às próprias características da espécie e mesmo a influência das condições meteorológicas, conforme citado por Lúcio et al. (2012).

Em relação ao comprimento do ramo principal, para o Ciclo 1, pôde-se observar o destaque da 'Abirú', com valores médios em torno de $111,3 \mathrm{~cm}(p<0,05)$, seguida da 'Tanzimech' (105,67 cm) e 'F1170' (97,7 cm) (Figura 3a). Neste sentido, embora se tenha verificado que a cv. Abirú apresentou valores mais elevados do diâmetro do caule e do comprimento do ramo, sobressaindo-se quanto às demais, não foram constatadas diferenças estatísticas destes dois parâmetros entre as cultivares analisadas $(p>0,05)$. As condições mais amenas observadas no Ciclo 2 favoreceram o crescimento do tomateiro para todas as cultivares analisadas, onde observou-se que a 'Tanzimech' se sobressaiu, com valores médios em torno de $214,00 \mathrm{~cm}$, seguida da 'Abirú' (164,33 cm), 'E5663' (140,33 cm) e 'F1170' (139,67 $\mathrm{cm})$ (Figura 3b), havendo diferenças estatísticas. Silva Filho et al. (2011), trabalhando com alguns parâmetros biométricos para análise do crescimento do tomate cereja 'Prati Pak', conduzido por tutoramento, observaram que o cultivo em casa de vegetação apresentou uma altura média (comprimento do ramo) em torno de $102,79 \mathrm{~cm}$.

Quando se analisou o número de folhas totalmente expandidas para o Ciclo 1, pôde-se observar que inicialmente as três cultivares apresentaram valores semelhantes, com aproximadamente 5 folhas cada, no entanto o mesmo não aconteceu para o Ciclo 2, sendo que as quatro cultivares 


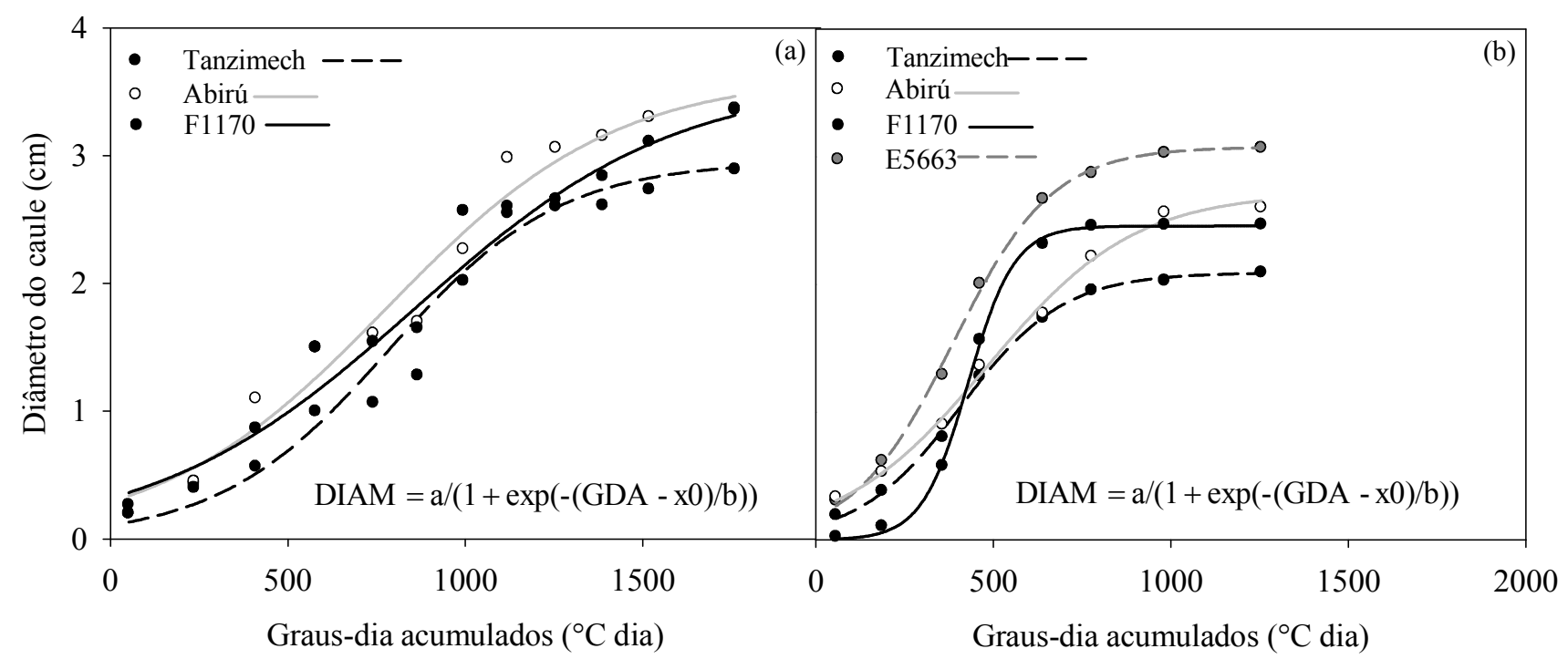

Figura 2. Diâmetro do caule (DIAM) para cultivares de tomate cereja, em função dos graus-dia acumulados (GDA), no Submédio do Vale do São Francisco: (a) Ciclo 1 e (b) Ciclo 2.

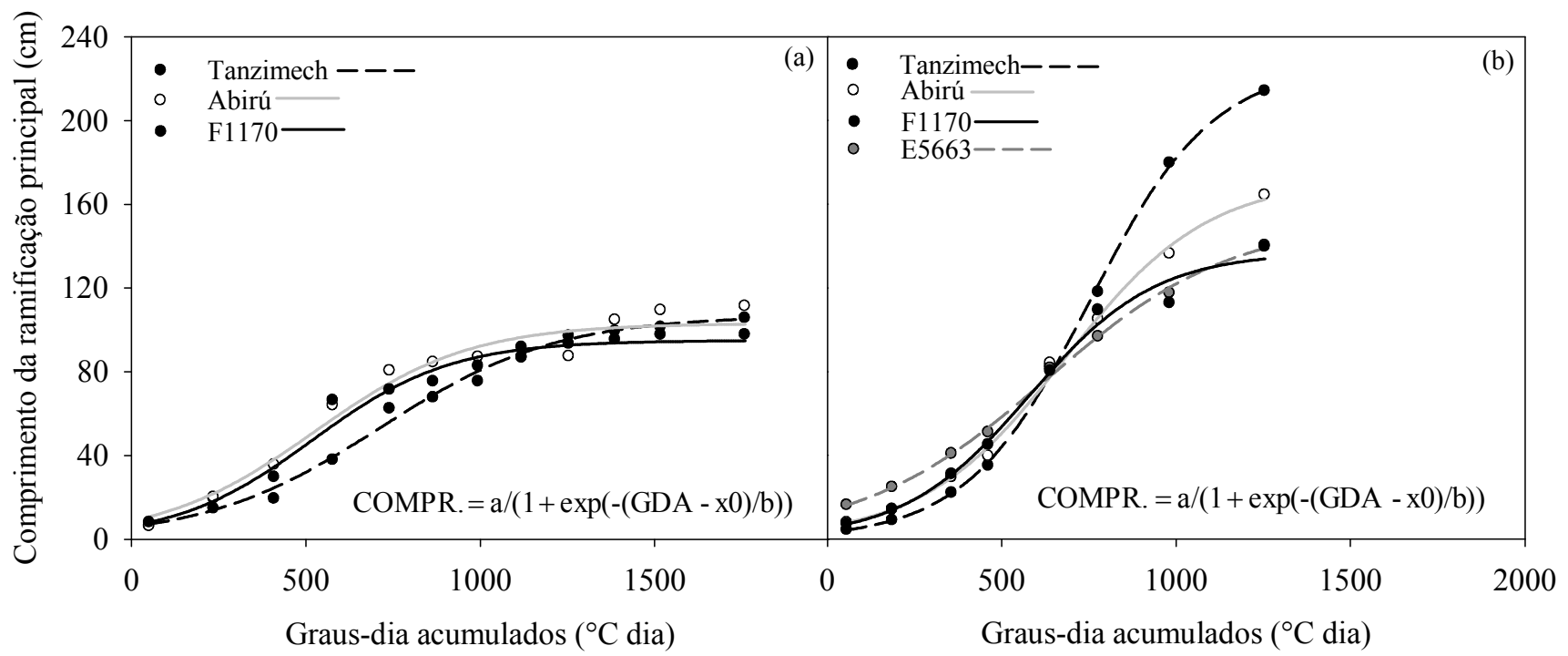

Figura 3. Comprimento da ramificação principal (COMPR.) de cultivares de tomate cereja, em função dos graus-dia acumulados (GDA), no Submédio Vale do São Francisco: (a) Ciclo 1 e (b) Ciclo 2.

apresentaram inicialmente valores entre 5 e 12 folhas. Posteriormente, as mesmas passam por uma fase de crescimento rápido, quando então se torna perceptível uma discrepância entre os valores do número de folhas da 'Tanzimech' ( $620 \pm 57$ folhas) quando comparada às cultivares Abirú (730 \pm 49 folhas) e F1170 ( $840 \pm 44$ folhas) no Ciclo 1 (Figura 4a). Já na segunda época analisada, observou-se uma diferença nos valores do número de folhas da 'F1170' ( $810 \pm 47$ folhas) e 'E5663' ( $820 \pm 59$ folhas) quando comparada às cvs. Abirú (1000 \pm 164 folhas) e Tanzimech (1129 \pm 100 folhas) no Ciclo 2 (Figura $4 b$ ).

A relação do número de folhas e os graus-dia acumulados (GDA) resultaram na taxa de aparecimento foliar (TAF) de $0,44,0,51$ e 0,58 folhas ( $\left.{ }^{\circ} \mathrm{C} \mathrm{dia}\right)^{-1}$ para as variedades Tanzimech, Abirú e F1170, correspondentes a um filocrono de
$2,29,1,95$ e $1,74{ }^{\circ} \mathrm{C}$ dia folha ${ }^{-1}$, respectivamente, durante o Ciclo 1. A taxa de aparecimento foliar (TAF) do Ciclo 2 foi de $1,18,1,06,0,84$ e 0,85 folhas $\left({ }^{\circ} \mathrm{C} \text { dia) }\right)^{-1}$, respectivamente para as cvs. Tanzimech, Abirú, F1170 e E5663, correspondentes a um filocrono de $0,85,0,94,1,20$ e $1,18^{\circ} \mathrm{C}$ dia folha ${ }^{-1}$.

\section{Eficiência do uso da água}

Os dados de produção observados no Ciclo 1 e Ciclo 2 demonstram que mesmo em condições ambientais distintas, as cultivares de tomate cereja mantiveram a hierarquia produtiva, tendo destaque a 'E5663' (40,30 t ha'-1), seguida da 'F1170' (22,69 tha-1), 'Abirú' (18,45 tha-1) e a 'Tanzimech' $\left(8,72 \mathrm{t} \mathrm{ha}^{-1}\right)$ no Ciclo 1 (Tabela 1). Neste caso, as diferenças observadas podem ser atribuídas às características inerentes à própria cultivar como, por exemplo, as observadas no 


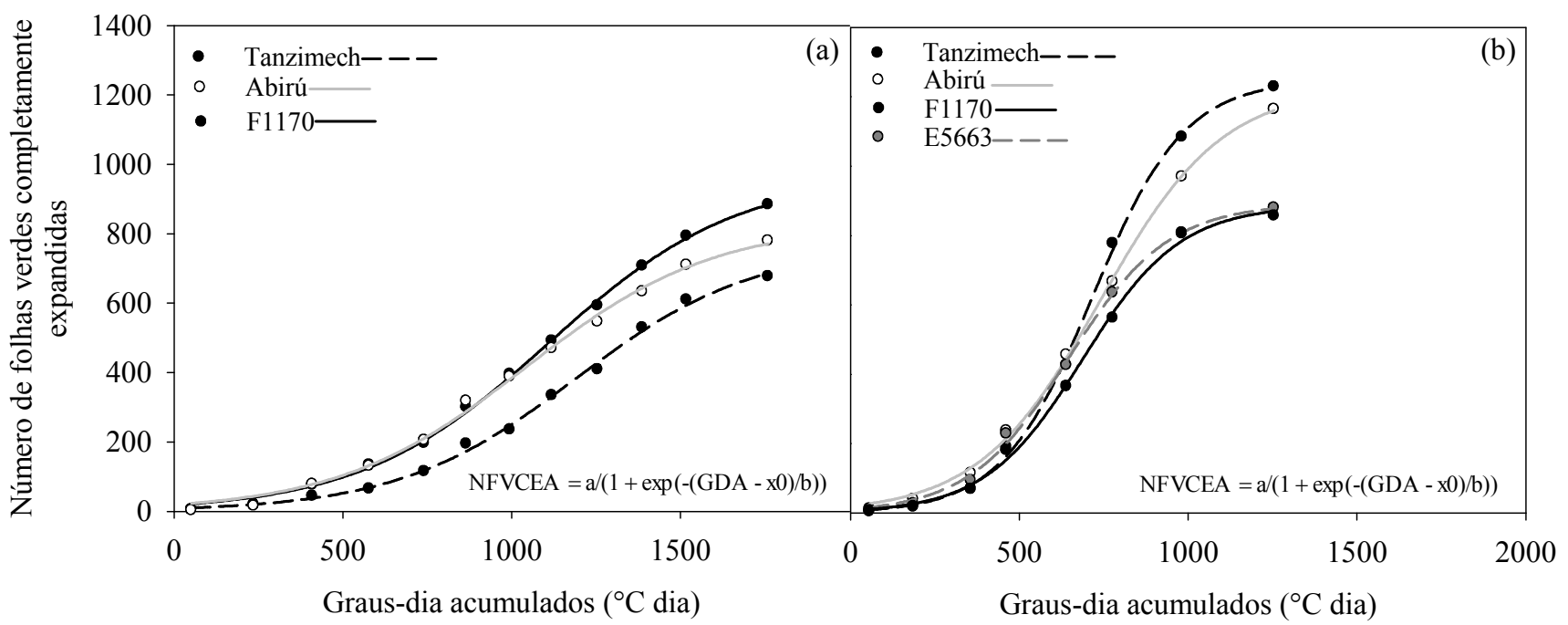

Figura 4. Número de folhas completamente expandidas (NFVCEA) para cultivares de tomate cereja, em função dos graus-dia acumulados (GDA), no Submédio Vale do São Francisco: (a) Ciclo 1 e (b) Ciclo 2.

número total e na morfologia das folhas, que é órgão responsável pela síntese de assimilados da cultura, bem como no formato e tamanho dos frutos.

No Ciclo 2, sob temperatura do ar mais amena quando comparada ao Ciclo 1 , foi observado que a produtividade (PF) das culturas praticamente dobrou. A cv. E5663 produziu 75,09 t ha' ${ }^{-1}$, seguida da 'Abirú' (61,09 t ha' $\left.{ }^{-1}\right)$, 'F1170' $\left(54,43 \mathrm{t} \mathrm{ha}^{-1}\right)$ e 'Tanzimech' (22,23 $\left.\mathrm{t} \mathrm{ha}^{-1}\right)$ (Tabela 1). Neste sentido, embora a cv. Tanzimech tenha apresentado um crescimento superior à 'E5663', devido à grande quans tidade de folhas e tamanho da planta, provavelmente, a maior parte dos assimilados produzidos foram utilizados para sua manutenção em detrimento aos frutos. Quando se estabeleceu uma comparação entre os dados de produção obtidos no presente estudo e os reportados na literatura, constatou-se que os mesmos foram superiores aos resultados observados por Silva et al. (2008), que analisando o tomate cereja, híbrido cv. Super-Sweet, sob as condições meteorologicas da região Seropédica, RJ, obtiveram produção igual a 16,12 tha ${ }^{1-}$. Porém, são inferiores aos resultados reportados por Maia (2012), que em estudo realizado em Viçosa, MG, realizado para analisar o cultivo do tomate cereja hidropônico, obteve uma média equivalente a 125,45 t ha ${ }^{-1}$, o que neste caso especifico pode estar associado ao emprego de uma maior densidade de plantio (31.347 plantas ha ${ }^{-1}$ e condições meteorológicas locais mais favoráveis à obtenção de melhores rendimentos, bem como pelo próprio sistema de cultivo hidropônico.

Ao se relacionar os dados de produção e a lamina de água aplicada observou-se que a produtividade de água da cultura (PAC) foi significativamente diferente para as cultivares analisadas nos dois ciclos $(p<0,05)$, mesmo não tendo sido constatadas diferenças entre as lâminas de água aplicadas (262,14 e 215,21 mm, para o Ciclo 1 e Ciclo
2, respectivamente). Em ambos os casos, observou-se que a 'E5663' apresentou maior desempenho na utilização da água em relação às demais, com uma diferença de produção de até $80 \%$ e $71 \%$ quando comparada a cultivar menos produtiva, que em ambas as épocas foi a 'Tanzimech' (Tabela 1). Neste caso, em se tratando de uma região com características semiáridas, como as observadas no presente estudo, a identificação de cultivares que apresentem uma maior produtividade, com uma maximização no uso da água é de extrema importância. Trabalhos que relatem a eficiência do uso de água para o tomate cereja são escassos, especialmente em condições semiáridas. Abrahão, Villlas Bôas, Bull (2014) verificaram diferentes eficiências de uso da água nas cultivares de tomate cereja, sendo que a 'Swett Milion' apresentou 29,1 $\mathrm{kg} \mathrm{m}^{-3} \mathrm{e}$ a 'Sweet Grape' 22,3 $\mathrm{kg} \mathrm{m}^{-3}$. Já Malheiros et al. (2012) analisando a cultivar Dellycia, nas condições litorâneas do Estado de Pernambuco, observaram que os ganhos produtivos poderiam atingir até $31,3 \mathrm{~kg}$ $\mathrm{m}^{-3}$ com uso de $50 \%$ de efluentes tratado de uma indústria de sorvete. Em todos os casos as divergências encontradas entre os valores de eficiência do uso da água podem ser atribuídos às cultivares estudadas, as condições climáticas locais e ao espaçamento de plantio. Menezes et al. (2012) verificaram que em cultivo de verão, o tomate plantado em Montes Claros, MG, apresentou produtividades da ordem de 11,95 $\mathrm{t} \mathrm{ha}^{-1}$, com destaque para a 'Carolina' com 14,4 t ha $^{-1}$.

Quando se analisou o ganho econômico das cultivares, obtido pela relação entre a produtividade média $\left(t h \mathrm{~h}^{-1}\right) \mathrm{e}$ o preço médio comercial do quilograma de tomate, $R \$ 6,41$ (valor médio de sete anos), constatou-se uma maior viabilidade no cultivo da cv. E5663. Por outro lado, a 'F1170' e a 'Abirú' também apresentaram bons resultados e não se diferiram estatisticamente em termos de ganho bruto 
Tabela 1. Indicadores de eficiência do uso da água para cultivares de tomate cereja em duas épocas distintas nas condições climáticas do Semiárido brasileiro.

\begin{tabular}{lllll}
\hline Cultivar & PF $\left(\mathbf{t ~ h a}^{-1}\right)$ & PAC $\left(\mathbf{k g ~ m}^{-\mathbf{3}}\right)$ & GE $\left(\mathbf{R} \$ \mathbf{~ h a}^{-1}\right)$ & PEA $\left(\mathbf{R \$ ~ ~ ^ { - 3 } )}\right.$ \\
\hline Tanzimech & $8,72 \mathrm{a}$ & $3,60 \mathrm{a}$ & $55,87 \mathrm{a}$ & $23,08 \mathrm{a}$ \\
F1170 & $22,69 \mathrm{~b}$ & $12,62 \mathrm{~b}$ & $145,46 \mathrm{ab}$ & $80,87 \mathrm{~b}$ \\
Abirú & $18,45 \mathrm{ab}$ & $11,69 \mathrm{~b}$ & $118,26 \mathrm{~b}$ & $74,93 \mathrm{~b}$ \\
E5663 & $40,30 \mathrm{~b}$ & $15,37 \mathrm{~b}$ & $258,32 \mathrm{~b}$ & $98,54 \mathrm{~b}$ \\
\hline CV (\%) & 25,62 & 27,24 & 25,61 & 27,25 \\
\hline Média & 16,61 & 9,30 & 106,53 & 59,63 \\
\hline
\end{tabular}

Ciclo 2

\begin{tabular}{lllll}
\hline Tanzimech & $22,23 \mathrm{a}$ & $10,86 \mathrm{a}$ & $142,54 \mathrm{a}$ & $69,60 \mathrm{a}$ \\
F1170 & $54,43 \mathrm{ab}$ & $27,27 \mathrm{ab}$ & $348,89 \mathrm{ab}$ & $127,5 \mathrm{ab}$ \\
Abirú & $61,09 \mathrm{~b}$ & $30,35 \mathrm{~b}$ & $391,59 \mathrm{~b}$ & $194,5 \mathrm{ab}$ \\
E5663 & $75,09 \mathrm{~b}$ & $37,89 \mathrm{~b}$ & $481,31 \mathrm{~b}$ & $242,88 \mathrm{~b}$ \\
\hline CV $(\%)$ & 24,53 & 23,88 & 24,53 & 36,12 \\
\hline Média & 53,22 & 26,59 & 341,08 & 158,65 \\
\hline
\end{tabular}

$\mathrm{PF}=$ Produtividade de Frutos, PAC $=$ Produtividade de Água da Cultura, PEA $=$ Produtividade Econômica de Água, GE = Ganho econômico.

(Tabela 1). No caso da produtividade econômica de água (PEA), tem-se ainda que os valores oscilaram entre 23,08 e $98,54 \mathrm{R} \$ \mathrm{~m}^{-3}$ para as variedades de tomate cereja no Ciclo 1 e entre 69,60 e 242,88 $\mathrm{R} \$ \mathrm{~m}^{-3}$ no Ciclo 2. Isto indica que no caso da E5663 retorna em média R\$170,72 para cada $1 \mathrm{~m}^{3}$ de água que entra no sistema via irrigação, enquanto a variedade Tanzimech apresentou retorno de $\mathrm{R} \$ 46,34$.

\section{Conclusões}

A cv. Tanzimech apresentou maior crescimento relacionado às características biométricas quando comparada às demais cultivares no ciclo 2 sob condições irrigadas semiáridas. Em termos produtivos a cv. E5663 apresentou melhor desempenho, que se refletiu tanto na eficiência do uso da água como no retorno econômico bruto, o que sugere a indicação de seu cultivo.

Devido à sazonalidade climática da região semiárida, constatou-se que o melhor desempenho produtivo das cultivares ocorreu quando o ciclo produtivo do tomate se desenvolve na época de condições meteorológicas mais amenas, entre março e julho.

\section{Referências}

ABRAHÃO, C., VILLAS BÔAS, R. L., BULL, L. T. Relação K:Ca:Mg na solução nutritiva para a produção de minitomate cultivado em substrato. Irriga, Botucatu, v. 19, n. 2, p. 214-224, 2014.

AGUIRRE, N. C., CABRERA, F. A. V. Evaluating the Fruit Production and Quality of Cherry Tomato (Solanum lycopersicum var. cerasiforme). Revista Facultad Nacional de Agronomía, Medellín, v. 65, n. 2, p. 65936604, 2012.

ALVARENGA, M.A.R. Tomate: produção em campo, em casa de vegetação e em hidroponia. Lavras: Editora UFLA, 2004. 400 p.

ANDRIOLO, J. L. Fisiologia da produção de hortaliças em ambiente protegido. Horticultura Brasileira, Brasília, v. 18, p. 26-33, 2000. Suplemento.

ARAÚJO, L. et al. Tomate cereja cultivado em diferentes concentrações de solução nutritiva no sistema hidropônico capilar. Revista Unimontes Científica, Montes Claros, v. 15, n.1, p. 18-27, 2013.

CARVALHO, L. A.; TESSARIOLI NETO, J. Produtividade de tomate em ambiente protegido, em função do espaçamento e número de ramos por planta. Horticultura Brasileira, Brasília, v.23, n. 4, p. 986-989, 2005.

CAVALCANTE, F. J. A. (coord.). Recomendações de adubação para o estado de Pernambuco: $2^{\mathrm{a}}$ aproximação. Recife: IPA, 2008. 212 p.

CEASA-BA. Disponível em: http://www.ebal.ba.gov.br/novagestao/ Ceasa_numeros_arquivos.htm. Acesso em: 22 Out, 2013.

EMBRAPA SEMIÁRIDO. Médias Anuais da Estação Agrometeorológica de Bebedouro. Disponível em: http://www.cpatsa.embrapa.br:8080/ servicos/dadosmet/ceb-anual.html. Acesso em: 17 Jan, 2013. 
GEERTS, S.; RAES, D. Deficit irrigation as an on-farm strategy to maximize crop water productivity in dry areas. Agricultural Water Management, Amsterdam, v. 96, p. 1275-1284, 2009.

GOMES JUNIOR, J. et al. Crescimento e produtividade de tomateiros do grupo cereja em função da aplicação de biofertilizante líquido e fungo micorrízico arbuscular. Revista Brasileira de Ciências Agrárias, Recife, v. 6, n. 4, 2011, p. 627-633, Universidade Federal Rural de Pernambuco.

GUSMÃO, M. T. A.; GUSMÃO, S. A. L.; ARAÚJO, J. A. C. Produtividade de tomate tipo cereja cultivado em ambiente protegido e em diferentes substratos. Horticultura Brasileira, Brasília, v.24, p. 431-436, 2006.

KUMAR, R. et al. Partitioning of dry matter during drought stress in rainfed lowland rice. Field Crops Research, Amsterdam, v.96, p. 455465, 2006.

LACERDA, C. F. et al. Eficiência de utilização de água e nutrientes em plantas de feijão-de-corda irrigadas com água salina em diferentes estádios de desenvolvimento. Engenharia Agrícola, Botucatu, v.29, p.221-230, 2009.

LENUCCI, M. et al. Antioxidant composition in cherry and high-pigment tomato cultivars. Journal of Agriculture Food and Chemistry, Easton, v. 54, p. 2606-2613, 2006.

LEONARDI, C. et al. Antioxidative activity and carotenoid and tomatine contents in different typologies of fresh consumption tomatoes. Journal of Agricultural and Food Chemistry, Easton, v. 48, n. 10, p. 4723 -4727. 2000.

LIU, F.; STÜTZEL, H. Biomass partitioning, specific leaf area, and water use efficiency of vegetable amaranth (Amaranthus spp.) in response to drought stress. Scientia Horticulturae, Amsterdam, v. 102, p. 15-27, 2004 .

LÚCIO, A. D. et al. Tamanhos de amostra e de parcela para variáveis de crescimento e produtivas de tomateiro. Horticultura Brasileira, Brasília, v. 30, p. 660-668. 2012.

MAIA, J.T.L.S. Cultivo hidropônico do tomateiro do grupo cereja: Crescimento, produção e qualidade sob doses de K e sintomas visuais e anatomia sob omissão de nutrientes. Dissertação (Doutorado) - Universidade Federal de Viçosa, 2012.

MALHEIROS, S. M. M. et al. Cultivo hidropônico de tomate cereja utilizando-se efluente tratado de uma indústria de sorvete. Revista Brasileira de Engenharia Agrícola e Ambiental, Campina Grande, v.16, n.10, p.1085-1092, 2012.

MENEZES et al. Fruit production and classification of four cherry tomato genotypes under an organic cropping system. IDESIA, Arica, v. 30, n. 3, p. 29-35, 2012.
OLIVEIRA, A. R. et al. Absorção de nutrientes e resposta à adubação em linhagens de tomateiro. Hortilcultura Brasileira, Brasília, v. 27, p. 498$504,2009$.

OMETTO, J. C. Bioclimatologia vegetal. São Paulo: Agronômica Ceres $440.1981 \mathrm{p}$.

PAYERO, J. O. et al. Effect of irrigation amounts applied with subsurface drip irrigation on corn evapotranspiration, yield, water use efficiency, and dry matter production in a semiarid climate. Agricultural Water Management, Amsterdam, v.8, p. 895-908, 2008.

PEREIRA, L.S.; CORDERY, I.; IACOVOS, I. Improved indicators of water use peformance and productivity for sustainable water conservation and saving. Agricultural Water Management, Amsterdam, v.108, p.39-51. 2012.

PUPPALA, N. et al. Evapotranspiration, yield, and water-use efficiency responses of Lesquerella fendleri at different growth stages. Industrial Crops and Products. v.21, p.33-47. 2005.

RADIN, B. et al. Eficiência de uso da radiação fotossinteticamente ativa pela cultura do tomateiro em diferentes ambientes. Pesquisa Agropecuária Brasileira, Brasília, v. 38, n. 9, p. 1017-1023, 2003.

SARI, B. G. et al. Linear relationships between cherry tomato traits. Ciência Rural, Santa Maria, v. 47, n. 03, p. 1-7, 2017.

SILVA FILHO, J. B. et al. Crescimento de tomate cereja em diferentes volumes de solução nutritiva no sistema de hidroponia capilar com TNT. Horticultura Brasileira, Brasília, v. 29, p. 2489-S2497. 2011.

SILVA, P. R. A. et al. Avaliação de acessos de tomate cereja para cultivo em sistema orgânico de produção. Evaluation of cherry tomatoes access on organic production system. Horticultura Brasileira, Brasília, v. 26, p. 2393-S2398. 2008.

SOUZA, J. F.; MEDEIROS, M. J. M.; CARNEIRO, L. C. Caracterização de Tomates (Lycopersicon Esculentum), Cultivar 'Cerejas' Produzidos e Comercializados nos Estados do Ceará e Rio Grande do Norte. In: V Congresso Norte - Nordeste de Pesquisa e Inovação da Rede Norte e Nordeste de Educação Tecnológica, 2010, Maceió - AL. Anais 2010, 2010.

TEIXEIRA, A. H. de C. Informações agrometeorológicas do Polo Petrolina, PE/Juazeiro, BA - 1963 a 2009. Petrolina: Embrapa Semiárido, 2010. 21 p. (Embrapa Semiárido. Documentos, 233).

REFERENCIAÇÃO

MOURA, M. S. B. de; SOUZA, L. S. B. de; OLIVEIRA, L. D. da S.; SILVA, T. G. F. da; YURI, J. E. Biometria e eficiência do uso da água em tomate cereja no semiárido. Agrometeoros, Passo Fundo, v.25, n.1, p.175-183, 2017.

Declaração: os trabalhos estão sendo publicados nesse número de AGROMETEOROS (v.25, n.1, ago 2017) conforme foram aceitos pelo XX Congresso Brasileiro de Agrometeorologia, realizado de 14 a 18 de agosto de 2017, em Juazeiro, BA e Petrolina, PE, sem revisão editorial adicional da revista. 


\title{
Biometrics and efficiency of water use in cherry tomato in semiarid
}

\author{
Magna Soelma Beserra de Moura ${ }^{1(*)}$, Luciana Sandra Bastos de Souza ${ }^{2}$, Leide Dayane da Silva Oliveira ${ }^{3}$, \\ Thieres George Freire da Silva ${ }^{2}$, Jony Eishi Yuri ${ }^{1}$ \\ ${ }^{1}$ Pesquisador(a), Embrapa Semiárido, Petrolina, PE, magna.moura@embrapa.br, jony.yuri@embrapa.br \\ ${ }^{2}$ Professor(a), UFRPE/UAST, Serra Talhada, PE, sanddrabastos@hotmail.com, thigeoprofissional@hotmail.com \\ ${ }^{3}$ Bolsista BFT/FACEPE, Embrapa Semiárido, Petrolina, PE, leide_dayane2014@hotmail.com \\ ${ }^{(*)}$ Corresponding author
}

\section{ARTICLE INFO}

Article history:

Recebido em 16 de Junho de 2017

Aceito em 10 de agosto de 2017

Index terms:

growth

productivity

water requirement

Solanum lycopersicum.

\section{ABSTRACT}

The objective of this study was to evaluate the biometric and efficiency of water use in tomato in semiarid region. The experiment was conducted at two times of the year, with cycles began in September 12, 2012 and April 15, 2013, in the experimental field of Embrapa Semiárido, municipally of Petrolina, State of Pernambuco, Brazil. The cultivars evaluated were the F1170, Abirú, Tanzimech and E5663, in which the biometric monitoring measures to stem diameter, length of the main stem and number of expanded leaves, throughout the cycle, and at the time of the harvest was measured yield. In the analysis of the efficiency of water use was used the indicators: crop water productivity (CWP, $\mathrm{kg} \mathrm{m}^{-3}$ ) and the economic water productivity (EWP, $\mathrm{R} \$ \mathrm{~m}^{-3}$ ). The results showed that, in the second planting season (April 15, 2013), the growth of cherry tomato was superior to the cv. Tanzimech $(214.00 \mathrm{~cm})(\mathrm{p}<0.05)$. However, the highest yield (75.1 ton ha-1), water use efficiency $\left(37.89 \mathrm{Kg} \mathrm{m}^{3-}\right)$ and water economic productivity $\left(242.88 \mathrm{R} \$ \mathrm{~m}^{-3}\right)$ were to the $\mathrm{cv}$. E5663, suggesting that its cultivation is encouraged under the conditions of the Low-middle São Francisco River Valley.

Disclaimer: papers are published in this issue of AGROMETEOROS (v. 25, n.1, aug 2017) as accepted by the XX Congresso Brasileiro de Agrometeorologia, held August 14-18, 2017 in Juazeiro, Bahia and Petrolina, Pernambuco, Brazil, without further revision by editorial board. 